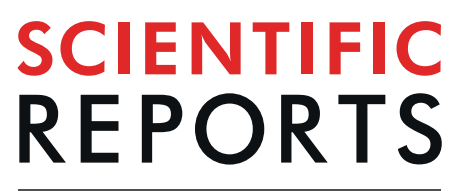

natureresearch

\title{
OPEN Low FVC/TLC in Preserved Ratio Impaired Spirometry (PRISm) is associated with features of and progression to obstructive lung disease
}

\begin{abstract}
Spyridon Fortis ${ }^{1,2^{*}}$, Alejandro Comellas ${ }^{2}$, Victor $\mathrm{Kim}^{3}$, Richard Casaburi ${ }^{4}$, John E. Hokanson ${ }^{5}$, James D. Crapo ${ }^{6}$, Edwin K. Silverman ${ }^{7}$ \& Emily S. Wan ${ }^{7,8}$

One quarter of individuals with Preserved Ratio Impaired Spirometry (PRISm) will develop airflow obstruction, but there are no established methods to identify these individuals. We examined the utility of FVC/TLC in identifying features of obstructive lung disease. The ratio of post-bronchodilator FVC and $T_{L E} C_{C T}$ from chest $C T$ (FVC/TLC $C$ ) among current and former smokers with PRISm (FEV $/$ /FVC $\geq 0.7$ and FEV1 $<80 \%$ ) in COPDGene was used to stratify subjects into quartiles: very high, high, low, and very low. We examined the associations between $\mathrm{FVC/TLC} \mathrm{CT}_{\mathrm{CT}}$ quartiles and (1) baseline characteristics, (2) respiratory exacerbations, (3) progression to COPD at 5 years, and (4) all-cause mortality. Among participants with PRISm at baseline $(n=1,131)$, the very low $\mathrm{FVC} / T L C_{\mathrm{CT}}$ quartile was associated with increased gas trapping and emphysema, and higher rates of progression to COPD at 5 years ( $36 \%$ versus $17 \% ; p<0.001$ ) relative to the very high quartile. The very low $\mathrm{FVC} / \mathrm{TLC}_{\mathrm{CT}}$ quartile was associated with increased total (IRR $=1.65 ; 95 \% \mathrm{Cl}[1.07-2.54])$ and severe (IRR $=2.24 ; 95 \% \mathrm{Cl}[1.29-3.89])$ respiratory exacerbations. Mortality was lower in the very high $\mathrm{FVC/TLC} \mathrm{CT}_{\mathrm{C}}$ quartile relative to the other quartiles combined. Reduced FVC/TLC $\mathrm{CT}_{\mathrm{T}}$ ratio in PRISm is associated with increased symptoms, radiographic emphysema and gas trapping, exacerbations, and progression to COPD.
\end{abstract}

Among individuals in the general population with at least 10 pack-years of cigarette smoking who undergo spirometry, $12 \%$ have a post-bronchodilator $\mathrm{FEV}_{1} \%$ predicted below $80 \%$ with a $\mathrm{FEV}_{1} / \mathrm{FVC}$ ratio $\geq 0.7^{1}$. This "non-specific" spirometric pattern, commonly referred to as "restrictive", has been designated as Preserved Ratio Impaired Spirometry (PRISm) ${ }^{2-7}$. Smokers with PRISm have higher cumulative smoking exposure, reduced exercise capacity, and increased mortality compared to smokers with normal lung function ${ }^{1,2,7}$. PRISm is also associated with thicker airway walls relative to smokers with normal spirometry ${ }^{2}$. PRISm individuals share features with COPD patients, and a quarter of subjects with PRISm eventually develop COPD ${ }^{1,2}$. The range of post-bronchodilator $\mathrm{FEV}_{1} \%$ predicted and percent emphysema on CT among PRISm subjects is wide, indicative of a heterogeneous population ${ }^{2}$. Clustering analysis identified a "PRISm-COPD" subgroup with lower FEV 1 /FVC ratio and higher radiologic CT emphysema relative to the rest of PRISm subjects ${ }^{3}$. This subgroup may represent individuals with early or occult obstructive lung disease who do not meet current diagnostic thresholds for airflow limitation.

\footnotetext{
${ }^{1}$ Center for Access \& Delivery Research \& Evaluation (CADRE), lowa City VA Health Care System, lowa City, IA, USA. ${ }^{2}$ Division of Pulmonary, Critical Care and Occupational Medicine, University of lowa Hospital and Clinics, lowa City, IA, USA. ${ }^{3}$ Department of Thoracic Medicine and Surgery, Lewis Katz School of Medicine at Temple University, Philadelphia, PA, USA. 'Lundquist Institute for Biomedical Innovation at Harbor-UCLA Medical Center, Torrance, CA, USA. ${ }^{5}$ Department of Epidemiology, Colorado School of Public Health, University of Colorado Denver, Denver, CO, USA. ${ }^{6}$ Department of Medicine, National Jewish Health, Denver, CO, USA. ${ }^{7}$ Channing Division of Network Medicine, Brigham and Women's Hospital, Boston, MA, USA. ${ }^{8}$ VA Boston Healthcare System, Jamaica Plain, MA, USA. *email: spyridon-fortis@uiowa.edu
} 
In PRISm, total lung capacity (TLC) may help distinguish a restrictive from an obstructive ventilatory defect, according to the American Thoracic Society-European Respiratory Society (ATS- ERS) 2005 guidelines ${ }^{8}$. However, a true "restrictive disease" is very unlikely in individuals with risk factors for obstruction lung disease, no interstitial lung disease and unremarkable body mass index (BMI). In COPDGene, the prevalence of PRISm is $12 \%$ despite the fact that participants with interstitial lung disease were excluded and body mass index (BMI) in PRISm individuals was slightly higher than the BMI in smokers with normal lung function ${ }^{1,2}$. In addition, a single center study showed that among individuals with PRISm and TLC above the lower limit of normal (LLN), only $26 \%$ had a clinical diagnosis of obstructive lung disease ${ }^{9}$. Moreover, only $15 \%$ of those with PRISm and TLC > LLN develop obstructive spirometry over a median follow-up time of 3 years ${ }^{10}$. Currently, there is no available diagnostic test in clinical practice to identify which patients with PRISm may have features classically associated with obstructive lung disease.

In obstructive lung diseases, residual volume (RV) may increase at the expense of FVC with total lung capacity (TLC) remaining normal ${ }^{11}$. Conversely, RV may increase with a preserved FVC resulting in an increased TLC. Both processes result in reduced FVC/TLC ratio which may antedate the development of obstruction diagnosed using standard $\mathrm{FEV}_{1} / \mathrm{FVC}$ criteria. A disproportionate decrease of $\mathrm{FVC}$ relative to TLC may occur in patients with a restrictive ventilatory defect that coexists with obstructive lung disease ${ }^{12}$. Thus, FVC/TLC represents a composite measure that may be able to identify an occult obstructive ventilatory defect. FVC can be readily obtained from spirometry, while TLC, typically assessed by plethysmography, can also be quantified using an inspiratory chest $\mathrm{CT}\left(\mathrm{TLC}_{\mathrm{CT}}\right)$, with prior studies demonstrating strong correlations with the plethysmography results ${ }^{13,14}$.

We hypothesized that reduced FVC/TLC ratio in PRISm is associated with clinical, functional and radiographic features of obstructive lung disease, acute respiratory events and increased mortality, and progression to COPD. To investigate our hypothesis, we examined current and former smokers with PRISm enrolled in the COPDGene study.

\section{Methods}

Data collection. We analyzed data from participants in the COPDGene study, an ongoing study conducted at multiple clinical centers through the United States (http://www.copdgene.org/). Participants were current and former smokers with $\geq 10$ pack-years of smoking who self-identified as non-Hispanic whites (NHW) or African Americans (AA) and were between the ages of 45-80 years at enrollment. The institutional review boards at each participating center approved the study protocol and written informed consent was obtained from all participants. Details of the study protocol have been published previously ${ }^{15}$. Briefly, participants completed a modified American Thoracic Society Respiratory Epidemiology questionnaire, St. George's Respiratory Questionnaire (SGRQ), and 6-minute walk test (6-MWT) at the enrollment visit. Dyspnea was assessed using the modified Medical Research Council (mMRC) scale. Participants performed pre- and post-bronchodilator spirometry. The complete study protocols were performed in accordance with the relevant guidelines and regulations of American Thoracic Society-European Respiratory Society (ATS-ERS) ${ }^{16}$. Volumetric chest CT scans were obtained at TLC CT (maximal inspiration) and at functional residual capacity $\left(\mathrm{FRC}_{\mathrm{CT}}\right)$ (end-tidal expiration) using multidetector CT scanners ${ }^{15}$. FRC and TLC\% predicted were calculated based on the predicted values ${ }^{17}$. Percent emphysema and gas trapping were quantified using 3D Slicer software (www.airwayinspector.org) $)^{15}$.

We included participants with PRISm at enrollment. We excluded individuals with significant interstitial lung disease or bronchiectasis on chest CT, those with missing post-bronchodilator spirometry or TLC $\mathrm{CT}_{\text {measure- }}$ ments at baseline, and participants with post-bronchodilator $\mathrm{FVC}>\mathrm{TLC}_{\mathrm{CT}}$ at enrollment. Approximately 5 years after the enrollment visit, participants were invited for a follow-up visit that included a repeat spirometry and chest CT. Respiratory exacerbation data were collected prospectively after enrollment. Participants were contacted every 6 months after enrollment and completed a standardized questionnaire regarding respiratory exacerbations through the Longitudinal Follow Up program. Vital status was also ascertained using information from the social security death index and the Longitudinal Follow Up program.

Definitions and outcomes. PRISm was defined as post-bronchodilator $\mathrm{FEV}_{1}<80 \%$ predicted and $\mathrm{FEV}_{1} /$ $\mathrm{FVC} \geq 0.7$. COPD was defined as post-bronchodilator $\mathrm{FEV}_{1} / \mathrm{FVC}<0.7$. The $\mathrm{FVC} / \mathrm{TLC}_{\mathrm{CT}}$ ratio at enrollment was calculated using post-bronchodilator FVC (in liters) from spirometry, while $\mathrm{TLC}_{\mathrm{CT}}$ was measured from volumetric inspiratory chest CT scans.

Co-morbidities and medication usage were self-reported. Percent emphysema was defined by using the percentage of lung volume at $\mathrm{TLC}_{\mathrm{CT}}$ with attenuation less than -950 Hounsfield units $(\mathrm{HU})^{15}$. Gas trapping was quantified as the percentage of lung volume at FRC with attenuation values less than $-856 \mathrm{HU}^{15}$. Parametric response mapping analysis was performed on paired registered inspiratory and expiratory images to distinguish functional small airways disease $\left(\mathrm{PRM}^{\mathrm{fSAD}}\right.$ ) from emphysema by Imbio LLC (Minneapolis, MN) using lung density analysis software ${ }^{18}$. As previous described ${ }^{19}$, we defined $\mathrm{PRM}^{\mathrm{fSAD}}$ as the percentage of lung with evidence of gas trapping not due to emphysema (i.e. areas of lung with attenuation $<-856 \mathrm{HU}$ on expiration minus area of lung with attenuation $<-950 \mathrm{HU}$ on inspiration).

Change in $\mathrm{FEV}_{1}$ between enrollment and 5-year follow up visit was calculated using post-bronchodilator spirometry. Exacerbations were defined as episodes of worsening respiratory symptoms requiring use of antibiotics and/or systemic steroids. Severe exacerbations were defined as those requiring hospitalizations or emergency room visits. Other variable definitions have been previously described ${ }^{15}$.

Statistical analysis. We stratified PRISm participants at the enrollment visit into quartiles by FVC/TLC $\mathrm{CT}_{\text {: }}$ very high, high, low, and very low. We compared the characteristics of PRISm individuals at the enrollment visit, rates of progression to COPD at the 5-year follow-up visit, and exacerbations over the time between the $\mathrm{FVC} / \mathrm{TLC}_{\mathrm{CT}}$ quartiles. We used Spearman's rank correlation to examine changes in continuous variables with 
increasing $\mathrm{FVC} / \mathrm{TLC}_{\mathrm{CT}}$. We used the Cochran Armitage trend test to examine proportion changes with increasing FVC/TLC ${ }_{\mathrm{CT}}$ quartile.

We created multivariable logistic and linear regression models with chronic bronchitis, mMRC and SGRQ scores, radiographic measures and 6-MWT distance at the enrollment visit as the dependent variable (outcome) and post-bronchodilator $\mathrm{FVC} / \mathrm{TLC}_{\mathrm{CT}}$ quartile at the enrollment as the independent variable (predictor). All models included the following covariates: age and current smoking status at enrollment, gender, race, pack-years smoked, body mass index (BMI), history of asthma and congestive heart failure. There were no missing values in any of the covariates. We also performed a multivariable linear and logistic regression analysis with change in $\mathrm{FEV}_{1}, 6-\mathrm{MWT}$ distance, radiographic measurements, and progression to COPD at the follow-up visit as the dependent variable (outcome) and post-bronchodilator $\mathrm{FVC}_{\mathrm{TLC}}$ (T) quartile at enrollment as the independent variable (predictor). We included the following covariates in these models: age and current smoking status at enrollment, gender, race, pack-years smoked, body mass index (BMI), history of asthma and congestive heart failure, and change of BMI between enrollment and follow-up visit.

For the exacerbation analysis, we created zero-inflated negative binomial models which included adjustment for age and current smoking status at enrollment, gender, race, pack-years smoked, BMI, chronic bronchitis, history of asthma and congestive heart failure. There were no missing values in any of the covariates. Follow-up time was included as an offset in the models as previously described ${ }^{20}$.

We used Cox proportional hazard regression models to examine the association between post-bronchodilator FVC/TLC $\mathrm{CT}_{\mathrm{C}}$ quartile with all-cause mortality. Models included the following covariates: age, gender, race, smoking status, smoking pack-years, BMI, diabetes, history of asthma and congestive heart failure. There were no missing values in any of the covariates.

In sensitivity analyses, we repeated selected analyses using PRISm defined as post-bronchodilator FEV1 $<$ LLN with a post-bronchodilator FEV1/FVC $\geq$ LLN and COPD defined as post-bronchodilator FEV1/ $\mathrm{FVC}<\mathrm{LLN}$ using the NHANES III reference values ${ }^{21}$. All statistical analyses were conducted using R statistical software (http://www.r-project.org/) using the following R software packages: 'dunn.test', 'FSA', 'pscl', 'MASS', 'AER', 'survival', and 'DescTools'.

Ethics approval. The institutional review boards at each participating center approved the study protocol. Details of the study protocol have been published previously ${ }^{16}$.

\section{Results}

Of 10,199 COPDGene participants with at least 10 or more pack-years of smoking and no significant interstitial lung disease or bronchiectasis, 1,260 of them had PRISm at the enrollment visit. After excluding one individual with no available post-bronchodilator spirometry, 121 with no TLC $_{\mathrm{CT}}$ measures and 7 individuals with $\mathrm{FVC}>\mathrm{TLC}_{\mathrm{CT}}, 1,131$ participants were included in the analysis. The median value of $\mathrm{FVC} / \mathrm{TLC}_{\mathrm{CT}}$ was 0.59 $(\mathrm{IQR}=0.53-0.66)$. Of these 1,131 participants, 617 of them had acceptable spirometry measurements at the 5-year follow-up visit, 967 had available data regarding respiratory exacerbations, and 960 had vital status data available.

Baseline characteristics at the enrollment visit $(n=1,131)$. Table 1 shows the characteristics of participants by FVC/TLC ${ }_{\mathrm{CT}}$ quartile. Age, BMI, pack-years smoking exposure, mMRC and SGRC scores, \% emphysema and gas trapping, and \% functional small airways disease increase with decreasing FVC/TLC $\mathrm{CT}_{\text {. An }}$ increased proportion of females and decreased proportion of African Americans were associated with decreasing $\mathrm{FVC}_{\text {TLC }}$ CT. Participants in the lower FVC/TLC $\mathrm{CT}_{\mathrm{C}}$ quartiles a higher prevalence of comorbidities.

In multivariable-adjusted analyses, the very low $\mathrm{FVC}_{\mathrm{TLC}} \mathrm{CT}_{\mathrm{C}}$ quartile was associated with an average of $3.31 \%$ higher radiographic gas trapping $(95 \% \mathrm{CI}=1.85-4.76 ; \mathrm{p}<0.001)$, and $3.26 \%$ higher $\mathrm{PRM}^{\mathrm{fSAD}}(95 \% \mathrm{CI}=1.40-$ 5.12; $\mathrm{p}<0.001$ ) relative to the very high quartile (Fig. 1). Lower quartiles were also associated with higher $\%$ emphysema. The very low quartile was associated with a trend towards higher SGRQ $(3.63 ; 95 \% \mathrm{CI}=-0.17$ to 7.44; $\mathrm{p}=0.06$ ) (Supplementary Table S1).

Progression to COPD at 5-year follow-up. Among participants with valid spirometry at the 5-year follow up visit $(n=617)$, approximately $35.9 \%$ (56 of 156$)$ of individuals in very low FVC/TLC ${ }_{\mathrm{CT}}$ quartile progressed to COPD, while 23\% (37 of 160), 22\% (35 of 156), and 17\% (25 of 145) of individuals in the low, high, and very high $\mathrm{FVC} / \mathrm{TLC}_{\mathrm{CT}}$ quartiles, respectively, progressed to COPD (Fig. 2; Cochran-Armitage test for trend $\mathrm{p}<0.001$ ). In the multivariable-adjusted analysis, the very low $\mathrm{FVC} / \mathrm{TLC}_{\mathrm{CT}}$ quartile at enrollment was associated with progression to COPD with an OR of $2.67(95 \% \mathrm{CI}=1.45-5.00 ; \mathrm{p}<0.001)$ relative to the highest quartile (Supplementary Table S2).

Longitudinal changes in spirometry, functional capacity, and radiographic features. Supplement Table S2 shows changes in spirometry, functional capacity, and radiographic features between enrollment and follow-up visit. In the adjusted analysis, the very low FVC/TLC $\mathrm{CT}$ quartile at enrollment was associated with increase of $2.74 \%$ radiographic gas trapping $(95 \% \mathrm{CI}=0.55-4.93 ; \mathrm{p}=0.014)$ relative to the highest quartile (Supplementary Table S3). FVC/TLC $\mathrm{CT}_{\mathrm{C}}$ was not associated with change in FEV1, 6-MWT distance or \% emphysema over time. There were no differences in the rate of decline in FEV1 by current smoking status at enrollment (combined and by FVC/TLC quartile - data not shown).

Respiratory exacerbations. Of 967 subjects with exacerbation data available, 349 (36.1\%) reported at least one exacerbation and $196(20.3 \%)$ reported at least one severe exacerbation during a median follow-up time of 6.4 years ( $\mathrm{IQR}=3.8$ to 7.4 ). Approximately, 44\% (115 of 262), 37\% (93 of 250), 31\% (72 of 232), and $31 \%$ (69 of 223) in the very low, low, high, and very high Quartiles had at least one exacerbation during the time period (Cochran-Armitage trend test $\mathrm{p}<0.001)$. In the very low, low, high, and very high Quartiles, 26\% (67 of 262), 


\begin{tabular}{|c|c|c|c|c|c|}
\hline \multirow[b]{2}{*}{ FVC/TLC Quartile } & \multicolumn{4}{|c|}{ High air trapping $\rightarrow$ Low air trapping } & \multirow[b]{2}{*}{$\begin{array}{l}\text { P for } \\
\text { trend }\end{array}$} \\
\hline & $\begin{array}{l}\text { Very Low } \\
\text { quartile }(n=283)\end{array}$ & $\begin{array}{l}\text { Low quartile } \\
(\mathbf{n}=\mathbf{2 8 3})\end{array}$ & $\begin{array}{l}\text { High quartile } \\
(n=282)\end{array}$ & $\begin{array}{l}\text { Very High } \\
\text { quartile }(n=283)\end{array}$ & \\
\hline $\mathrm{FVC/TLC} \mathrm{CT}_{\mathrm{CT}}$ & $<0.53$ & $0.53-0.59$ & $0.59-0.66$ & $>0.66$ & \\
\hline Age, $y \pm S D$ & $62.83 \pm 8.82$ & $57.68 \pm 7.34$ & $55.70 \pm 6.96)$ & $52.84 \pm 6.27$ & $<0.001$ \\
\hline Female, $\mathbf{n}(\%)$ & $186(65.7 \%)$ & $169(59.7 \%)$ & $143(50.7 \%)$ & $115(40.6 \%)$ & $<0.001$ \\
\hline African American, n (\%) & $86(30.4 \%)$ & $89(31.4 \%)$ & $126(44.7 \%)$ & $174(61.5 \%)$ & $<0.001$ \\
\hline $\mathrm{BMI}, \mathrm{Kg} / \mathrm{m}^{2} \pm \mathrm{SD}$ & $32.99 \pm 7.42$ & $32.84 \pm 7.50$ & $30.24 \pm 6.88$ & $31.05 \pm 6.97$ & $<0.001$ \\
\hline Pack-Years \pm SD & $49.46 \pm 28.69$ & $43.51 \pm 22.51$ & $39.12 \pm 20.08$ & $38.06 \pm 22.63$ & $<0.001$ \\
\hline Active Smoker, n (\%) & $154(54.4 \%)$ & $162(57.2 \%)$ & $180(63.8 \%)$ & $213(75.3 \%)$ & $<0.001$ \\
\hline Chronic Bronchitis, n (\%) & $53(18.7 \%)$ & $54(19.1 \%)$ & $52(18.4 \%)$ & $42(14.8 \%)$ & 0.23 \\
\hline $\mathrm{mMRC} \pm \mathrm{SD}$ & $1.70 \pm 1.47$ & $1.56 \pm 1.44$ & $1.21 \pm 1.37$ & $1.44 \pm 1.50$ & $<0.001$ \\
\hline SGRQ \pm SD & $32.91 \pm 22.69$ & $30.06 \pm 23.29$ & $24.50 \pm 20.54$ & $29.71 \pm 23.78$ & $<0.001$ \\
\hline Asthma, n (\%) & $75(26.5 \%)$ & $64(22.6 \%)$ & $51(18.1 \%)$ & $60(21.2 \%)$ & 0.064 \\
\hline CHF, n (\%) & $21(7.4 \%)$ & $17(6.0 \%)$ & $5(1.8 \%)$ & $8(2.8 \%)$ & 0.001 \\
\hline DM, n (\%) & $75(26.5 \%)$ & $75(26.5 \%)$ & $44(15.6 \%)$ & $42(14.8 \%)$ & $<0.001$ \\
\hline HTN, n (\%) & $150(53.0 \%)$ & $151(53.4 \%)$ & $133(47.2 \%)$ & $120(42.4 \%)$ & 0.004 \\
\hline CAD, n (\%) & $25(8.8 \%)$ & $32(11.3 \%)$ & $12(4.3 \%)$ & $10(3.5 \%)$ & $<0.001$ \\
\hline OSA, n (\%) & $68(24.0 \%)$ & $61(21.6 \%)$ & $56(19.9 \%)$ & $38(13.4 \%)$ & 0.002 \\
\hline CVA, n (\%) & $15(5.3 \%)$ & $10(3.5 \%)$ & $7(2.5 \%)$ & $7(2.5 \%)$ & 0.049 \\
\hline LAMA, n (\%) & $33(12.1 \%)$ & $18(6.5 \%)$ & $18(6.5 \%)$ & $15(5.4 \%)$ & 0.005 \\
\hline ICS, $n(\%)$ & $19(7.0 \%)$ & $19(6.8 \%)$ & $12(4.4 \%)$ & $13(4.6 \%)$ & 0.131 \\
\hline LABA, n (\%) & $7(2.6 \%)$ & $1(0.4 \%)$ & $1(0.4 \%)$ & $4(1.4 \%)$ & 0.2482 \\
\hline ICS/LABA, n (\%) & $59(21.6 \%)$ & $36(12.9 \%)$ & $22(7.9 \%)$ & $24(8.6 \%)$ & $<0.001$ \\
\hline Post-FEV1\% \pm SD & $65.74 \pm 9.65$ & $71.33 \pm 7.32$ & $72.04 \pm 6.54$ & $73.02 \pm 5.92$ & $<0.001$ \\
\hline Post-FVC $\% \pm$ SD & $66.70 \pm 10.07$ & $72.47 \pm 7.68$ & $73.47 \pm 7.58$ & $75.05 \pm 7.25$ & $<0.001$ \\
\hline BDR, n (\%) & $40(14.4 \%)$ & $41(14.6 \%)$ & $30(10.8 \%)$ & $47(16.8 \%)$ & 0.71 \\
\hline$\$ \%$ Emphysema \pm SD & $2.02 \pm 3.32$ & $1.66 \pm 2.92$ & $1.48 \pm 2.00$ & $1.07 \pm 1.53$ & $<0.001$ \\
\hline${ }^{\S} \%$ Gas trapping $\pm S D$ & $12.48 \pm 8.64$ & $9.06 \pm 7.48$ & $8.19 \pm 6.65$ & $7.50 \pm 5.82$ & $<0.001$ \\
\hline${ }^{7} \mathrm{PRM}^{\mathrm{fSAD}}, \% \pm \mathrm{SD}$ & $14.63 \pm 9.87$ & $10.60 \pm 8.65$ & $10.37 \pm 9.24$ & $10.13 \pm 8.65$ & $<0.001$ \\
\hline${ }^{5} \mathrm{FRC}_{\mathrm{CT}} \% \pm \mathrm{SD}$ & $97.25 \pm 18.17$ & $87.62 \pm 14.63$ & $80.89 \pm 13.00$ & $75.25 \pm 12.36$ & $<0.001$ \\
\hline $\mathrm{TLC}_{\mathrm{CT}} \% \pm \mathrm{SD}$ & $90.20 \pm 13.58$ & $85.82 \pm 9.80$ & $77.77 \pm 9.48$ & $68.96 \pm 9.02$ & $<0.001$ \\
\hline "6-MWT, meters \pm SD & $366.33 \pm 110.24$ & $394.00 \pm 104.72$ & $406.56 \pm 114.12$ & $396.23 \pm 109.78$ & $<0.001$ \\
\hline
\end{tabular}

Table 1. Baseline characteristics of smokers with preserved ratio impaired spirometry across postbronchodilator forced vital capacity/total lung capacity ratio $\left(\mathrm{FVC} / \mathrm{TLC}_{\mathrm{CT}}\right)$ quartiles $(\mathrm{n}=1,131)$. ${ }^{\varsigma}$ For \% GT and $\mathrm{FRC}_{\mathrm{CT}} \%$ analysis, data were available for 936 subjects. ${ }^{*}$ For PRM data analysis, data were available for 932 subjects. "For 6-MWT data analysis, data were available for 1,121 subjects. BDR = bronchodilator response; $\mathrm{BMI}=$ body mass index $\mathrm{CAD}=$ coronary artery disease; $\mathrm{CHF}=$ congestive heart failure; $\mathrm{DM}=$ diabetes mellitus; $\mathrm{FRC}_{\mathrm{CT}} \%=$ functional residual capacity $\%$ predicted; $\mathrm{HTN}=$ hypertension; $\mathrm{ICS}=$ inhaled glucocorticosteroids, LABA = long-acting beta-agonist, LAMA = long-acting muscarinic antagonist, mMRC $=$ modified Medical Research Council dyspnea score; OSA = obstructive sleep apnea; post-FEV $1 \%=$ postbronchodilator $\mathrm{FEV} 1 \%$ predicted; post- $\mathrm{FVC} \%=$ post-bronchodilator $\mathrm{FVC} \%$ predicted; $\mathrm{PRM}^{\mathrm{fSAD}}=$ parametric response mapping functional small airways disease; $\mathrm{SD}=$ standard deviation; $\mathrm{SGRQ}=$ St. George's Respiratory Questionnaire score; $\mathrm{TLC}_{\mathrm{CT}} \%=$ total lung capacity $\%$ predicted and 6-MWD $=6$-min walk test.

$18 \%$ (46 of 250), 16\% (38 of 232), and 20\% (45 of 223) of participants, respectively, had at least one severe respiratory exacerbation, with a trend towards significance (Cochran-Armitage $\mathrm{p}=0.095$ ). We created multivariable zero-inflated negative binomial models to examine the association of FVC/ TLC $_{\mathrm{CT}}$ quartile with respiratory exacerbations (Fig. 3). The very low FVC/TLC ${ }_{\mathrm{CT}}$ quartile was associated with increased relative risk for total exacerbations $(\mathrm{IRR}=1.65 ; 95 \% \mathrm{CI}=1.07-2.54 ; \mathrm{p}=0.023)$ and severe $(\mathrm{IRR}=2.24 ; 95 \% \mathrm{CI}=1.29-3.89 ; \mathrm{p}=0.004)$ exacerbations relative to the "very high" FVC/TLC quartile (Supplementary Table S4).

Mortality $(\mathbf{n}=\mathbf{9 6 0})$. During a median follow-up time of 2,408 days (IQR $=2158$ to 2622$), 12.9 \%$ (32 of 248) subjects died in the Very Low quartile, $11 \%$ (27 of 246) died in the low quartile, $11.3 \%$ (28 of 247) died in the High quartile, and $5.9 \%$ (13 of 219) died in the very high quartile (Cochran-Armitage trend test $\mathrm{p}=0.02)$. A Kaplan-Meier plot of mortality by FVC/TLC $\mathrm{CT}_{\mathrm{C}}$ quartile at enrollment is shown in Fig. 4.

In Cox proportional hazards models adjusted for age at enrollment, sex, race, BMI, current smoking at enrollment, cumulative smoking exposure, diabetes, history of asthma and congestive heart failure, increased mortality in the high quartile with a trends towards an increased mortality in the low and very low quartiles relative to the very high quartile was observed (Table 2). In a Cox proportional hazards model examining individuals in the very high quartile relative to all other quartiles (high, low, very low) combined, a reduced risk of mortality was observed $(\mathrm{HR}=0.53,95 \% \mathrm{CI}=0.28-0.97, \mathrm{p}=0.040)$. 
mMRC

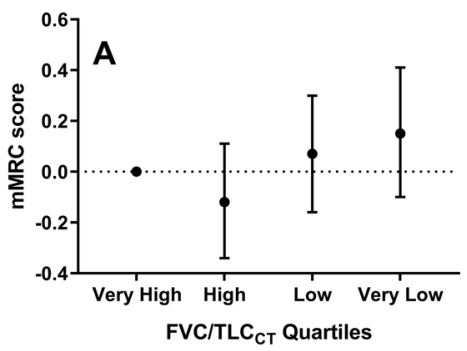

Emphysema $_{C T}$

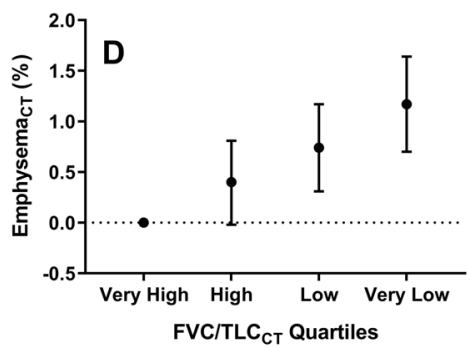

SGRQ

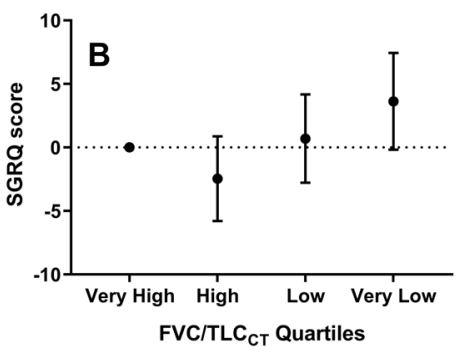

Gas Trapping $c T$

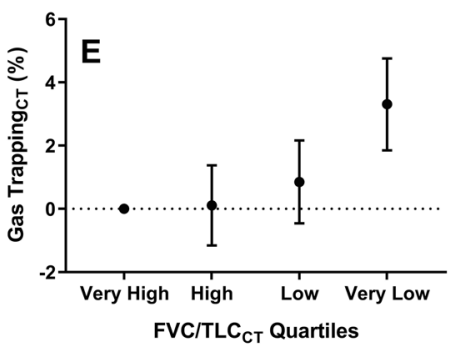

6-min walk distance

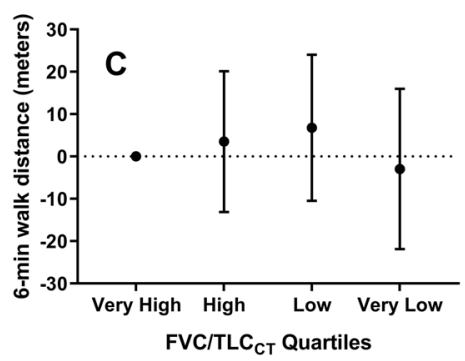

PRM ${ }^{\text {ISAD }}$

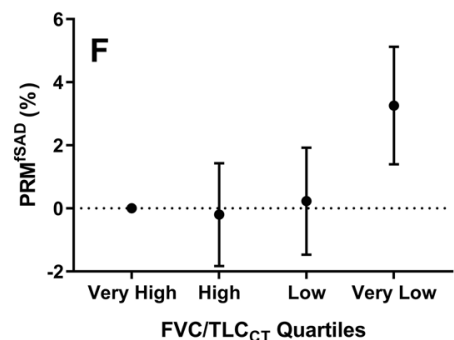

Figure 1. Associations between post-bronchodilator forced vital capacity/total lung capacity (FVC/TLC $\mathrm{CT}_{\text {) }}$ ) quartiles at enrollment with dyspnea and health-related quality of life scores, chest CT \% emphysema and \% gas trapping, functional small airway disease, and 6-min walk test distance at baseline among smokers with Preserved Ratio Impaired Spirometry (PRISm; $n=1,131$ ). Each panel in the figure represents a separate linear regression model with categorical post-bronchodilator $\mathrm{FVC}_{\mathrm{TLC}} \mathrm{CT}$ quartile as the main independent variable (exposure) with the "very high" quartile used as the reference category. The dependent variable (outcome) in each model was (A) modified Medical Research Council (mMRC) dyspnea score, (B) St. George's Respiratory Questionnaire total score (SGRQ), (C) 6-minute walk test distance (6-MWT in meters), (D) \% Emphysema, (E) $\%$ Gas trapping, and (F) functional small airways disease $\left(\mathrm{PRM}^{\mathrm{fSAD}}\right)$. All models were adjusted for the following co-variates: age, sex, race, smoking status, smoking pack-years, body mass index (BMI), history of asthma and congestive heart failure, and diabetes mellitus. FVC/TLC $\mathrm{CT}_{\mathrm{C}}$ quartile is plotted on the $\mathrm{x}$-axis while the regression coefficient (and 95\% CI) for each category is plotted on the $y$-axis. ${ }^{*}$ For $\%$ GT analysis, $n=936$ subjects. ${ }^{\dagger}$ For $\mathrm{PRM}^{\text {fsad }}$ data analysis, $\mathrm{n}=932$ subjects. ${ }^{\#}$ For 6-min walk test distance analysis, $\mathrm{n}=1,121$ subjects.

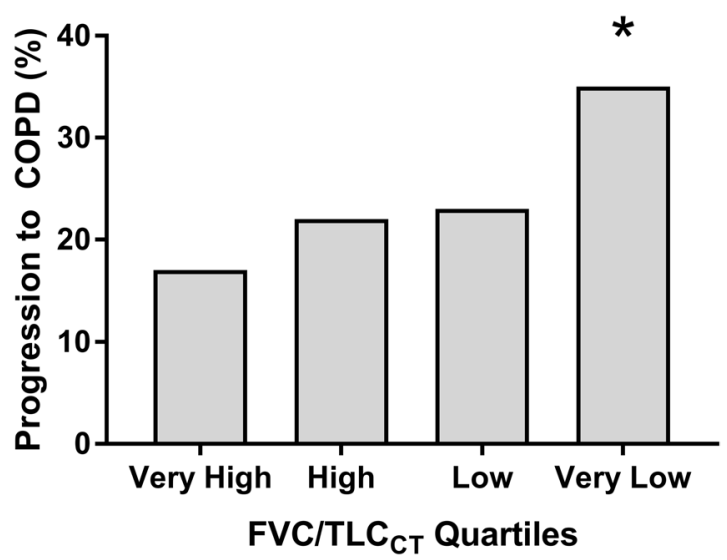

Figure 2. Progression to $\mathrm{COPD}\left(\mathrm{FEV}_{1} / \mathrm{FVC}<0.7\right)$ at the 5 -year follow-up visit by post-bronchodilator forced vital capacity/total lung capacity ratio $\left(\mathrm{FVC} / \mathrm{TLC}_{\mathrm{CT}}\right)$ quartiles at enrollment in smokers with preserved ratio impaired spirometry $(n=617)$. Cochran Armitage Trend test $\mathrm{p}<0.001$. Pairwise comparisons between quartiles performed using Chi-squared test. $* \mathrm{p}=0.026$ vs Very High FVC/TLC $\mathrm{CT}_{\mathrm{C}}$ Quartile.

Sensitivity analysis. When PRISm was defined using LLN criteria, we observed similar findings with those in main analysis except that $\mathrm{FVC} / \mathrm{TLC}_{\mathrm{CT}}$ was significantly associated with chronic bronchitis, increased $\mathrm{mMRC}$ and SGRQ and the association with mortality was attenuated (Supplementary Tables S5-S9 and Fig. S1). 


\section{Exacerbations}

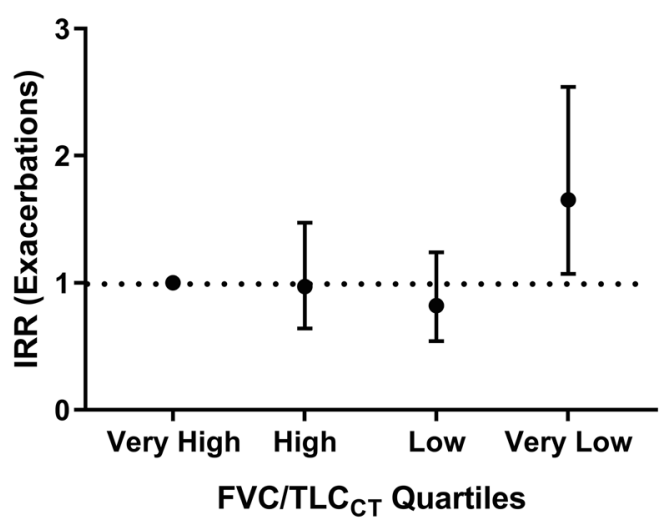

\section{Severe Exacerbations}

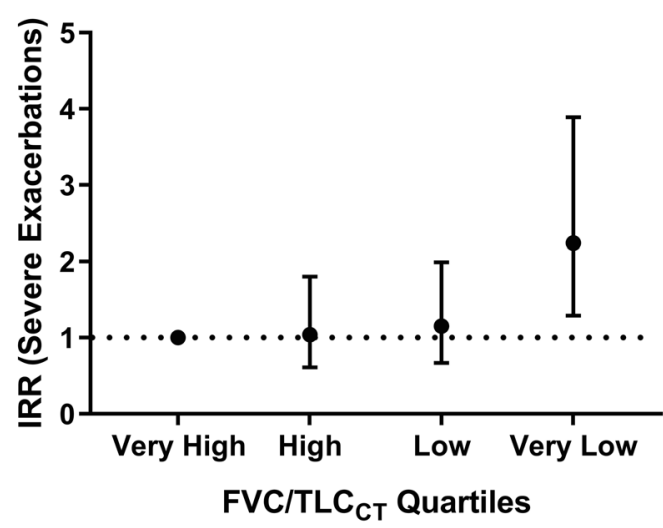

Figure 3. Associations between post-bronchodilator forced vital capacity/total lung capacity (FVC/TLC $\mathrm{CT}_{\text {T }}$ ) quartiles at enrollment with prospective exacerbations and severe exacerbations in smokers with Preserved Ratio Impaired Spirometry (PRISm; $n=967$ ). For exacerbation analysis, data for 967 subjects with PRISm at enrollment were available. Zero-inflated negative binomial regression models with post-bronchodilator FVC/ $\mathrm{TLC}_{\mathrm{CT}}$ as independent variable (exposure) and total exacerbations and severe exacerbations as the dependent variables (outcome) were performed. All regression models included the following co-variates: age, sex, race, body mass index, smoking status at the enrollment, smoking pack-years, history of asthma and congestive heart failure, and chronic bronchitis in the count negative binomial regression and an intercept-only model in the zero component. Follow-up time was included as an offset in the models. FVC/TLC ${ }_{\mathrm{CT}}$ quartile is plotted on the $\mathrm{x}$-axis while the IRR (and 95\% CI) for each category is plotted on the $\mathrm{y}$-axis. IRR = incidence rate ratio, $\mathrm{FVC} /$ $\mathrm{TLC}_{\mathrm{CT}}=$ forced vital capacity/total lung capacity.

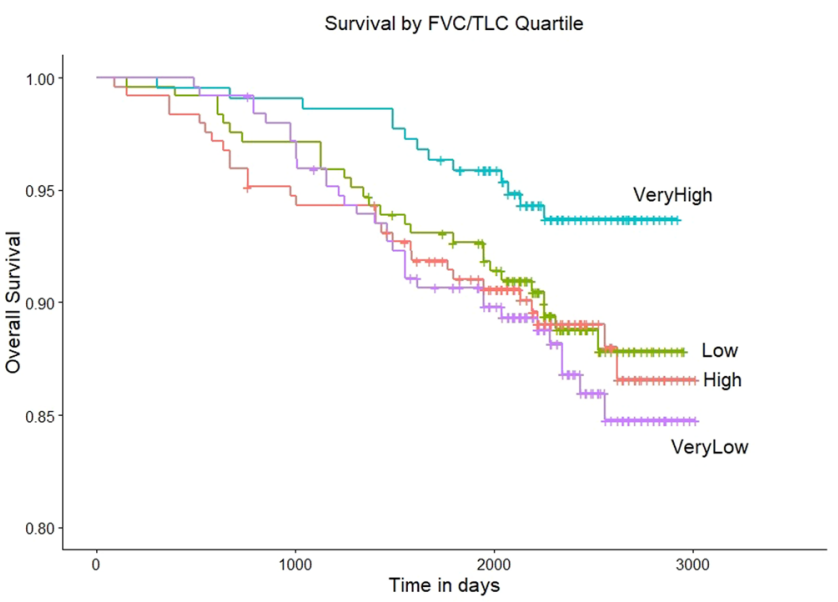

Figure 4. Kaplan-Meier Plot of overall survival by forced vital capacity/total lung capacity ratio (FVC/TLC $\left.{ }_{\mathrm{CT}}\right)$ quartiles at enrollment in smokers with Preserved Ratio Impaired Spirometry (PRISm, n=960). Chi-squared $\mathrm{p}$-value for differences in mortality by quartile $=0.07$.

\section{Discussion}

Our study explores the utility of $\mathrm{FVC} / \mathrm{TLC}_{\mathrm{CT}}$ ratio in former and current smokers with PRISm as a potential tool to identify individuals with features of and possible increased risk for progression to obstructive lung disease. In our cohort, very low $\mathrm{FVC}_{\text {TLC }}$, was associated with radiographic findings traditionally associated with COPD as well as progression to COPD and respiratory exacerbations while very high FVC/TLC $\mathrm{CT}_{\mathrm{C}}$ was associated with reduced mortality.

PRISm is a common spirometric pattern with a prevalence between $5 \%$ and $20 \%{ }^{22-25}$. Although often referred to as a "restrictive spirometric pattern", 30-40\% of patients with PRISm do not have reduced TLC ${ }^{26,27}$. On average, individuals PRISm have higher BMI, but obesity alone does not decrease vital capacity or TLC below the LLN in most individuals ${ }^{28}$. Notably, only about $5 \%$ of patients undergoing bariatric surgery for extreme obesity have PRISm at preoperative assessment ${ }^{29}$.

PRISm is comprised of a heterogeneous population with a wide range of BMI, degree of lung function impairment, and radiographic emphysema likely due to different underlying pathological processes in each individual ${ }^{2}$. Subgroups within PRISm may have increased risk for $\mathrm{FEV}_{1}$ decline, progression to COPD, exacerbations, and 


\begin{tabular}{|l|l|l|l|l|}
\hline \multirow{2}{*}{ Quartile } & \multicolumn{4}{|l|}{ FVC/TLC } \\
\cline { 2 - 5 } & HR & $\mathbf{2 . 5 \%}$ & $\mathbf{9 . 7 5} \%$ & P value \\
\hline Very High & ref & ref & ref & ref \\
\hline High & 2.12 & 1.09 & 4.13 & 0.028 \\
\hline Low & 1.68 & 0.84 & 3.36 & 0.14 \\
\hline Very Low & 1.87 & 0.89 & 3.87 & 0.10 \\
\hline
\end{tabular}

Table 2. Associations of post-bronchodilator forced vital capacity/total lung capacity (FVC/TLC $\mathrm{CT}_{\text {) }}$ quartiles at enrollment with mortality in smokers with Preserved Ratio Impaired Spirometry (PRISm; n = 960). Cox Hazard regression models with post-bronchodilator FVC/TLCCT quartiles as independent variables (exposure) and mortality as the dependent variable(outcome) were performed. All models for mortality included the following co-variates: age, sex, race, smoking status, smoking pack-years, body mass index (BMI), history of asthma and congestive heart failure, and diabetes mellitus. $\mathrm{HR}=$ Hazard Ratio.

mortality. In this manuscript, we utilize FVC/TLC, which decreases in obstructive lung disease ${ }^{11}$, as a conceptual surrogate for RV (which was not directly measured in our cohort) to identify individuals with features obstructive lung disease within PRISm. Our finding that individuals with PRISm with low FVC/TLC have increased radiographic emphysema and gas trapping complements work from the SPIROMICS cohort, where that RV/TLC was shown to be associated with increased radiographic emphysema and gas trapping in smokers with normal lung function $^{30}$. Apart from the fact that RV/TLC was not available in our cohort, we used FVC/TLC as it may be more sensitive to identify the presence of small airway disease than RV/TLC because FVC, a dynamic measure obtained at forced expiration, will capture dynamic collapse and air trapping not present during slow exhalation maneu$\operatorname{ver}^{31,32}$. Future studies should examine the role of RV/TLC in PRISm. In addition, we did not examine FRC/TLC as FRC can be reduced remarkably in obesity ${ }^{28}$ which may render difficult to interpret those measures when an obstructive lung diseases coexists.

Our findings suggest that low $\mathrm{FVC}_{\mathrm{TLC}}$ may be a possible a marker of early obstructive pulmonary disease. Nevertheless, participants in the very low FVC/TLC ${ }_{\mathrm{CT}}$ quartile have higher BMI; this contrasts with the common knowledge that patients with established obstructive pulmonary disease have often lower BMI. Previous studies have shown an inverse relationship of BMI with mortality in COPD, known also as the "obesity paradox" with confounders such as exercise capacity and muscle mass possibly contributing towards favorable outcomes ${ }^{33,34}$. In addition, despite the fact that obesity does not typically reduce the FVC below the LLN in subjects without lung disease ${ }^{29}$, higher BMI decreases FVC and increases the $\mathrm{FEV}_{1} / \mathrm{FVC}$ ratio which can lead to underdiagnosis of obstructive pulmonary disease ${ }^{31,35}$. In COPD subjects with established airflow obstruction, increasing BMI is associated with higher $\mathrm{FEV}_{1} / \mathrm{FVC}^{35}$.

We acknowledge that the fixed threshold FEV1/FVC $<0.7$ diagnostic criterion for COPD endorsed by Global Initiative for Chronic Obstructive Lung Disease(GOLD) may have also misclassified individuals with obstructive lung disease as PRISm. In a recent large population-based sample $(n=24,207)$, Bhatt and colleagues showed that the discriminative accuracy of $\mathrm{FEV}_{1} / \mathrm{FVC}<0.7$ to predict $\mathrm{COPD}$-related death and/or hospitalization was not inferior to $\mathrm{FEV}_{1} / \mathrm{FVC}<\mathrm{LLN}^{36}$. We assert that because the majority of our findings remained robust on sensitivity analyses using LLN-defined lung function categories. FVC/TLC ratio can be utilized to identify individuals with features of obstructive lung disease regardless of whether fixed-threshold or LLN criteria are used.

In COPDGene, $40.5 \%$ of PRISm individuals and $32.5 \%$ of smokers with normal lung function are African American ${ }^{2}$. Differences in the reliability of prediction equations may lead to the "overdiagnosis" of African American with PRISm in the absence of true pathology; this may contribute to the lower rates of African Americans in the low FVC/TLC $\mathrm{TT}_{\mathrm{T}}$ quartiles. It is also unclear why females were relatively over-represented in the lower FVC/TLC ${ }_{\mathrm{CT}}$ quartiles. Whether PRISm represents a gender-specific pathway to COPD, or whether traditional $\mathrm{FEV}_{1} / \mathrm{FVC}$ criteria systematically misclassify women with COPD is not known ${ }^{37,38}$. Our sensitivity analysis using gender and race specific spirometric criteria to define PRISm showed similar findings. Future studies, especially in cohorts of diverse ancestry and ethnicity, are warranted to further explore these findings.

Previous studies have shown that air trapping is associated with $\mathrm{FEV}_{1}$ decline. In current and former smokers with at least 20 pack-years smoking and normal lung function, RV/TLC is associated with $\mathrm{FEV}_{1}$ decline $^{30}$. We have extended these finding by showing that air trapping (low FVC/TLC) in individuals with PRISm is associated with progression to COPD. General population studies have also shown that individuals with abnormal non-obstructed lung function are at risk for developing COPD ${ }^{39,40}$. It may seem counterintuitive that low FVC/ $\mathrm{TLC}_{\mathrm{CT}}$ in PRISm was associated with progression to COPD and respiratory exacerbations, but was not associated with $\mathrm{FEV}_{1}$ decline, increase in emphysema, and change in 6-MWT distance over time ${ }^{41}$. Within COPDGene, individuals with PRISm are at increased risk for respiratory exacerbations relative to smokers with normal lung function $^{42}$. However, respiratory exacerbations in PRISm do not result in significant excess lung function decline in $\mathrm{FEV}_{1}$ as observed in individuals with established airflow limitation ${ }^{43}$. A survivor bias may also be present in FEV1 decline analysis ${ }^{43}$. Participants that had poor lung function and low FVC/TLC may have died before the follow-up visit. Similarly, we may have not observed changes in 6-MWT distance likely due to the high variability of the test ${ }^{44,45}$.

Population-based studies have shown that PRISm is associated with increased cardiac ${ }^{7}$ and all-cause mortality ${ }^{1,22}$. While the increased average BMI in PRISm as a whole may mediate some of the risk associated with increased mortality, the association between very high $\mathrm{FVC} / \mathrm{TLC} \mathrm{CT}_{\mathrm{C}}$ and lower mortality relative to all other quartiles despite concurrent adjustment for BMI, congestive heart failure, and diabetes status in our study suggests 
our composite measure may have utility in the risk-stratification of individuals with PRISm. Previous studies in COPD have shown associations between RV and mortality ${ }^{46}$. In Veterans with a history of smoking and normal lung function, Zeng et al. showed that RV/TLC is associated with increased respiratory medication use, hospitalizations, and all-cause mortality ${ }^{47}$.

Our study is the first one showing that a composite measure of lung function may help to identify patients with PRISm who eventually progress to classic airflow obstruction and are at increased risk for respiratory exacerbations and death. The strengths of our study include large sample size, highly granular epidemiological data, axial radiographic imaging data, and longitudinal data on clinically relevant outcomes. Despite this, we acknowledge the following limitations. RV was not available in our cohort. TLC $\mathrm{CT}_{\mathrm{T}}$ was measured in supine position by chest CT, which is usually lower than TLC measured in seated position by plethysmography ${ }^{13}$. Another limitation is possible self-selection bias of subjects who returned for a follow-up visit. Since our cohort includes only smokers, our findings cannot be generalized to non-smoking populations and future studies in independent cohorts are warranted. In conclusion, $\mathrm{FVC} / \mathrm{TLC}_{\mathrm{CT}}$ can help to identify individuals with PRISm at increased risk for clinical events and progression to COPD, and who would benefit from smoking cessation and may be a potential target population for treatment trials in the future.

Grant support and disclaimer. The project described was supported by Award Number U01 HL089897 and Award Number U01 HL089856 from the National Heart, Lung, and Blood Institute. The content is solely the responsibility of the authors and does not necessarily represent the official views of the National Heart, Lung, and Blood Institute or the National Institutes of Health. Department of Veterans Affairs, Veterans Health Administration, Office of Rural Health, Veterans Rural Health Resource Center (Award \# 14380), and the Health Services Research and Development (HSR\&D) Service through the Comprehensive Access and Delivery Research and Evaluation (CADRE) Center (CIN 13-412)U.S. Department of Veterans Affairs IK2RX002165. The views expressed in this article are those of the authors and do not necessarily reflect the position or policy of the Department of Veterans Affairs or the United States Government.

COPD foundation funding. The COPDGene project is also supported by the COPD Foundation through contributions made to an Industry Advisory Board comprised of AstraZeneca, Boehringer Ingelheim, GlaxoSmithKline, Novartis, Pfizer, Siemens and Sunovion.

COPDGene Investigators - core units. Administrative Center: James D. Crapo, MD (PI); Edwin K. Silverman, MD, PhD (PI); Barry J. Make, MD; Elizabeth A. Regan, MD, PhD.

Genetic Analysis Center: Terri Beaty, PhD; Ferdouse Begum, PhD; Peter J. Castaldi, MD, MSc; Michael Cho, MD; Dawn L. DeMeo, MD, MPH; Adel R. Boueiz, MD; Marilyn G. Foreman, MD, MS; Eitan Halper-Stromberg; Lystra P. Hayden, MD, MMSc; Craig P. Hersh, MD, MPH; Jacqueline Hetmanski, MS, MPH; Brian D. Hobbs, MD; John E. Hokanson, MPH, PhD; Nan Laird, PhD; Christoph Lange, PhD; Sharon M. Lutz, PhD; Merry-Lynn McDonald, PhD; Margaret M. Parker, PhD; Dandi Qiao, PhD; Elizabeth A. Regan, MD, PhD; Edwin K. Silverman, MD, PhD; Emily S. Wan, MD; Sungho Won, Ph.D.; Phuwanat Sakornsakolpat, M.D.; Dmitry Prokopenko, Ph.D. Imaging Center: Mustafa Al Qaisi, MD; Harvey O. Coxson, PhD; Teresa Gray; MeiLan K. Han, MD, MS; Eric A. Hoffman, PhD; Stephen Humphries, PhD; Francine L. Jacobson, MD, MPH; Philip F. Judy, PhD; Ella A. Kazerooni, MD; Alex Kluiber; David A. Lynch, MB; John D. Newell, Jr., MD; Elizabeth A. Regan, MD, PhD; James C. Ross, PhD; Raul San Jose Estepar, PhD; Joyce Schroeder, MD; Jered Sieren; Douglas Stinson; Berend C. Stoel, PhD; Juerg Tschirren, PhD; Edwin Van Beek, MD, PhD; Bram van Ginneken, PhD; Eva van Rikxoort, PhD; George Washko, MD; Carla G. Wilson, MS; PFT QA Center, Salt Lake City, UT: Robert Jensen, PhD.

Data Coordinating Center and Biostatistics, National Jewish Health, Denver, CO: Douglas Everett, PhD; Jim Crooks, PhD; Camille Moore, PhD; Matt Strand, PhD; Carla G. Wilson, MS.

Epidemiology Core, University of Colorado Anschutz Medical Campus, Aurora, CO: John E. Hokanson, MPH, PhD; John Hughes, PhD; Gregory Kinney, MPH, PhD; Sharon M. Lutz, PhD; Katherine Pratte, MSPH; Kendra A. Young, PhD.

Mortality Adjudication Core: Surya Bhatt, MD; Jessica Bon, MD; MeiLan K. Han, MD, MS; Barry Make, MD;

Carlos Martinez, MD, MS; Susan Murray, ScD; Elizabeth Regan, MD; Xavier Soler, MD; Carla G. Wilson, MS. Biomarker Core: Russell P. Bowler, MD, PhD; Katerina Kechris, PhD; Farnoush Banaei-Kashani, Ph.D.

COPDGene Investigators - clinical centers. Ann Arbor VA: Jeffrey L. Curtis, MD; Carlos H. Martinez, MD, MPH; Perry G. Pernicano, MD.

Baylor College of Medicine, Houston, TX: Nicola Hanania, MD, MS; Philip Alapat, MD; Mustafa Atik, MD; Venkata Bandi, MD; Aladin Boriek, PhD; Kalpatha Guntupalli, MD; Elizabeth Guy, MD; Arun Nachiappan, MD; Amit Parulekar, MD;

Brigham and Women's Hospital, Boston, MA: Dawn L. DeMeo, MD, MPH; Craig Hersh, MD, MPH; Francine L. Jacobson, MD, MPH; George Washko, MD.

Columbia University, New York, NY: R. Graham Barr, MD, DrPH; John Austin, MD; Belinda D’Souza, MD; Gregory D.N. Pearson, MD; Anna Rozenshtein, MD, MPH, FACR; Byron Thomashow, MD. Duke University Medical Center, Durham, NC: Neil MacIntyre, Jr., MD; H. Page McAdams, MD; Lacey Washington, MD.

HealthPartners Research Institute, Minneapolis, MN: Charlene McEvoy, MD, MPH; Joseph Tashjian, MD.

Johns Hopkins University, Baltimore, MD: Robert Wise, MD; Robert Brown, MD; Nadia N. Hansel, MD, MPH; Karen Horton, MD; Allison Lambert, MD, MHS; Nirupama Putcha, MD, MHS. 
Los Angeles Biomedical Research Institute at Harbor UCLA Medical Center, Torrance, CA: Richard Casaburi, $\mathrm{PhD}$, MD; Alessandra Adami, PhD; Matthew Budoff, MD; Hans Fischer, MD; Janos Porszasz, MD, PhD; Harry Rossiter, PhD; William Stringer, MD.

Michael E. DeBakey VAMC, Houston, TX: Amir Sharafkhaneh, MD, PhD; Charlie Lan, DO.

Minneapolis VA: Christine Wendt, MD; Brian Bell, MD.

Morehouse School of Medicine, Atlanta, GA: Marilyn G. Foreman, MD, MS; Eugene Berkowitz, MD, PhD; Gloria Westney, MD, MS

National Jewish Health, Denver, CO: Russell Bowler, MD, PhD; David A. Lynch, MB.

Reliant Medical Group, Worcester, MA: Richard Rosiello, MD; David Pace, MD.

Temple University, Philadelphia, PA: Gerard Criner, MD; David Ciccolella, MD; Francis Cordova, MD; Chandra Dass, MD; Gilbert D’Alonzo, DO; Parag Desai, MD; Michael Jacobs, PharmD; Steven Kelsen, MD, PhD; Victor Kim, MD; A. James Mamary, MD; Nathaniel Marchetti, DO; Aditi Satti, MD; Kartik Shenoy, MD; Robert M. Steiner, MD; Alex Swift, MD; Irene Swift, MD; Maria Elena Vega-Sanchez, MD.

University of Alabama, Birmingham, AL: Mark Dransfield, MD; William Bailey, MD; Surya Bhatt, MD; Anand Iyer, MD; Hrudaya Nath, MD; J. Michael Wells, MD.

University of California, San Diego, CA: Joe Ramsdell, MD; Paul Friedman, MD; Xavier Soler, MD, PhD; Andrew Yen, MD.

University of Iowa, Iowa City, IA: Alejandro P. Comellas, MD; Karin F. Hoth, PhD; John Newell, Jr., MD; Brad Thompson, MD.

University of Michigan, Ann Arbor, MI: MeiLan K. Han, MD, MS; Ella Kazerooni, MD; Carlos H. Martinez, $\mathrm{MD}, \mathrm{MPH}$

University of Minnesota, Minneapolis, MN: Joanne Billings, MD; Abbie Begnaud, MD; Tadashi Allen, MD.

University of Pittsburgh, Pittsburgh, PA: Frank Sciurba, MD; Jessica Bon, MD; Divay Chandra, MD, MSc; Carl Fuhrman, MD; Joel Weissfeld, MD, MPH.

University of Texas Health Science Center at San Antonio, San Antonio, TX: Antonio Anzueto, MD; Sandra Adams, MD; Diego Maselli-Caceres, MD; Mario E. Ruiz, MD.

\begin{tabular}{|c|c|c|}
\hline Clinical Center & Institution Title & Protocol Number \\
\hline National Jewish Health & National Jewish IRB & HS-1883a \\
\hline Brigham and Women's Hospital & Partners Human Research Committee & 2007-P-000554/2; BWH \\
\hline Baylor College of Medicine & $\begin{array}{l}\text { Institutional Review Board for Baylor College of } \\
\text { Medicine and Affiliated Hospitals }\end{array}$ & H-22209 \\
\hline Michael E. DeBakey VAMC & $\begin{array}{l}\text { Institutional Review Board for Baylor College of } \\
\text { Medicine and Affiliated Hospitals }\end{array}$ & H-22202 \\
\hline Columbia University Medical Center & Columbia University Medical Center IRB & IRB-AAAC9324 \\
\hline Duke University Medical Center & $\begin{array}{l}\text { The Duke University Health System Institutional } \\
\text { Review Board for Clinical Investigations (DUHS IRB) }\end{array}$ & Pro00004464 \\
\hline Johns Hopkins University & $\begin{array}{l}\text { Johns Hopkins Medicine Institutional Review Boards } \\
\text { (JHM IRB) }\end{array}$ & NA_00011524 \\
\hline Los Angeles Biomedical Research Institute & $\begin{array}{l}\text { The John F. Wolf, MD Human Subjects Committee of } \\
\text { Harbor-UCLA Medical Center }\end{array}$ & $12756-01$ \\
\hline Morehouse School of Medicine & $\begin{array}{l}\text { Morehouse School of Medicine Institutional Review } \\
\text { Board }\end{array}$ & $07-1029$ \\
\hline Temple University & $\begin{array}{l}\text { Temple University Office for Human Subjects } \\
\text { Protections Institutional Review Board }\end{array}$ & 11369 \\
\hline University of Alabama at Birmingham & $\begin{array}{l}\text { The University of Alabama at Birmingham } \\
\text { Institutional Review Board for Human Use }\end{array}$ & FO70712014 \\
\hline University of California, San Diego & $\begin{array}{l}\text { University of California, San Diego Human Research } \\
\text { Protections Program }\end{array}$ & 070876 \\
\hline University of Iowa & The University of Iowa Human Subjects Office & 200710717 \\
\hline Ann Arbor VA & VA Ann Arbor Healthcare System IRB & PCC 2008-110732 \\
\hline University of Minnesota & $\begin{array}{l}\text { University of Minnesota Research Subjects' Protection } \\
\text { Programs (RSPP) }\end{array}$ & $0801 \mathrm{M} 24949$ \\
\hline University of Pittsburgh & University of Pittsburgh Institutional Review Board & PRO07120059 \\
\hline $\begin{array}{l}\text { University of Texas Health Sciences Center } \\
\text { at San Antonio }\end{array}$ & $\begin{array}{l}\text { UT Health Science Center San Antonio Institutional } \\
\text { Review Board }\end{array}$ & HSC20070644H \\
\hline Health Partners Research Foundation & $\begin{array}{l}\text { Health Partners Research Foundation Institutional } \\
\text { Review Board }\end{array}$ & $07-127$ \\
\hline University of Michigan & Medical School Institutional Review Board (IRBMED) & HUM00014973 \\
\hline Minneapolis VA Medical Center & Minneapolis VAMC IRB & $4128-\mathrm{A}$ \\
\hline Fallon Clinic & $\begin{array}{l}\text { Institutional Review Board/Research Review } \\
\text { Committee Saint Vincent Hospital - Fallon Clinic - } \\
\text { Fallon Community Health Plan }\end{array}$ & 1143 \\
\hline
\end{tabular}

Received: 18 September 2019; Accepted: 17 February 2020;

Published online: 20 March 2020 


\section{References}

1. Wan, E. S. et al. Longitudinal Phenotypes and Mortality in Preserved Ratio Impaired Spirometry in the COPDGene Study. Am. J. Respir. Crit. Care Med. 198, 1397-1405, https://doi.org/10.1164/rccm.201804-0663OC (2018).

2. Wan, E. S. et al. Clinical and radiographic predictors of GOLD-unclassified smokers in the COPDGene study. Am. J. Respir. Crit. Care Med. 184, 57-63, https://doi.org/10.1164/rccm.201101-0021OC (2011).

3. Wan, E. S. et al. Epidemiology, genetics, and subtyping of preserved ratio impaired spirometry (PRISm) in COPDGene. Respir. Res. 15, 89, https://doi.org/10.1186/s12931-014-0089-y (2014).

4. Arne, M. et al. How often is diagnosis of COPD confirmed with spirometry? Respir. Med. 104, 550-556, https://doi.org/10.1016/j. rmed.2009.10.023 (2010).

5. Kohler, D., Fischer, J., Raschke, F. \& Schonhofer, B. Usefulness of GOLD classification of COPD severity. Thorax 58, 825, https://doi. org/10.1136/thorax.58.9.825 (2003).

6. Zielinski, J. et al. Increasing COPD awareness. Eur. Respir. J. 27, 833-852, https://doi.org/10.1183/09031936.06.00025905 (2006).

7. Guerra, S. et al. Morbidity and mortality associated with the restrictive spirometric pattern: a longitudinal study. Thorax $\mathbf{6 5}$, 499-504, https://doi.org/10.1136/thx.2009.126052 (2010).

8. Pellegrino, R. et al. Interpretative strategies for lung function tests. Eur. Respir. J. 26, 948-968, https://doi.org/10.1183/09031936.05. 00035205 (2005).

9. Hyatt, R. E., Cowl, C. T., Bjoraker, J. A. \& Scanlon, P. D. Conditions associated with an abnormal nonspecific pattern of pulmonary function tests. Chest 135, 419-424, https://doi.org/10.1378/chest.08-1235 (2009).

10. Iyer, V. N., Schroeder, D. R., Parker, K. O., Hyatt, R. E. \& Scanlon, P. D. The nonspecific pulmonary function test: longitudinal followup and outcomes. Chest 139, 878-886, https://doi.org/10.1378/chest.10-0804 (2011).

11. Ruppel, G. L. What is the clinical value of lung volumes? Respir. Care 57, 26-35; discussion 35-28, https://doi.org/10.4187/ respcare.01374 (2012).

12. Clay, R. D., Iyer, V. N., Reddy, D. R., Siontis, B. \& Scanlon, P. D. The “Complex Restrictive” Pulmonary Function Pattern: Clinical and Radiologic Analysis of a Common but Previously Undescribed Restrictive Pattern. Chest 152, 1258-1265, https://doi.org/10.1016/j. chest.2017.07.009 (2017).

13. O'Donnell, C. R. et al. Comparison of plethysmographic and helium dilution lung volumes: which is best for COPD? Chest 137, 1108-1115, https://doi.org/10.1378/chest.09-1504 (2010).

14. Iwano, S., Okada, T., Satake, H. \& Naganawa, S. 3D-CT volumetry of the lung using multidetector row CT: comparison with pulmonary function tests. Acad. Radiol. 16, 250-256, https://doi.org/10.1016/j.acra.2008.09.019 (2009).

15. Regan, E. A. et al. Genetic epidemiology of COPD (COPDGene) study design. COPD 7, 32-43, https://doi.org/10.3109/15412550903499522 (2010).

16. Miller, M. R. et al. Standardisation of spirometry. Eur. Respir. J. 26, 319-338, https://doi.org/10.1183/09031936.05.00034805 (2005).

17. Stocks, J. \& Quanjer, P. H. Reference values for residual volume, functional residual capacity and total lung capacity. ATS Workshop on Lung Volume Measurements. Official Statement of The European Respiratory Society. Eur. Respir. J. 8, 492-506, https://doi.org/ 10.1183/09031936.95.08030492 (1995).

18. Galban, C. J. et al. Computed tomography-based biomarker provides unique signature for diagnosis of COPD phenotypes and disease progression. Nat. Med. 18, 1711-1715, https://doi.org/10.1038/nm.2971 (2012).

19. Bhatt, S. P. et al. Association between Functional Small Airway Disease and FEV1 Decline in Chronic Obstructive Pulmonary Disease. Am. J. Respir. Crit. Care Med. 194, 178-184, https://doi.org/10.1164/rccm.201511-2219OC (2016).

20. Fortis, S. et al. Combined Forced Expiratory Volume in 1 Second and Forced Vital Capacity Bronchodilator Response, Exacerbations, and Mortality in Chronic Obstructive Pulmonary Disease. Ann. Am. Thorac. Soc. 16, 826-835, https://doi.org/10.1513/ AnnalsATS.201809-601OC (2019).

21. Hankinson, J. L., Odencrantz, J. R. \& Fedan, K. B. Spirometric reference values from a sample of the general U.S. population. Am. J. Respir. Crit. Care Med. 159, 179-187, https://doi.org/10.1164/ajrccm.159.1.9712108 (1999).

22. Mannino, D. M., Buist, A. S., Petty, T. L., Enright, P. L. \& Redd, S. C. Lung function and mortality in the United States: data from the First National Health and Nutrition Examination Survey follow up study. Thorax 58, 388-393, https://doi.org/10.1136/ thorax.58.5.388 (2003).

23. Mannino, D. M. et al. Restricted spirometry in the Burden of Lung Disease Study. Int. J. Tuberc. Lung Dis. 16, 1405-1411, https://doi. org/10.5588/ijtld.12.0054 (2012).

24. Backman, H. et al. Restrictive spirometric pattern in the general adult population: Methods of defining the condition and consequences on prevalence. Respir. Med. 120,116-123, https://doi.org/10.1016/j.rmed.2016.10.005 (2016).

25. Jankowich, M. et al. Restrictive Spirometry Pattern, Cardiac Structure and Function, and Incident Heart Failure in African Americans. The Jackson Heart Study. Ann. Am. Thorac. Soc. 15, 1186-1196, https://doi.org/10.1513/AnnalsATS.201803-184OC (2018).

26. Fortis, S., Corazalla, E. O., Jacobs, D. R. Jr. \& Kim, H. J. Persistent Empiric COPD Diagnosis and Treatment After Pulmonary Function Test Showed No Obstruction. Respir. Care 61, 1192-1200, https://doi.org/10.4187/respcare.04647 (2016).

27. Aaron, S. D., Dales, R. E. \& Cardinal, P. How accurate is spirometry at predicting restrictive pulmonary impairment? Chest 115, 869-873 (1999).

28. Jones, R. L. \& Nzekwu, M. M. The effects of body mass index on lung volumes. Chest 130, 827-833, https://doi.org/10.1378/ chest.130.3.827 (2006).

29. Clavellina-Gaytan, D. et al. Evaluation of spirometric testing as a routine preoperative assessment in patients undergoing bariatric surgery. Obes. Surg. 25, 530-536, https://doi.org/10.1007/s11695-014-1420-x (2015).

30. Arjomandi, M. et al. Radiographic lung volumes predict progression to COPD in smokers with preserved spirometry in SPIROMICS. Eur. Respir. J. 54, https://doi.org/10.1183/13993003.02214-2018 (2019).

31. Fortis, S., Corazalla, E. O., Wang, Q. \& Kim, H. J. The difference between slow and forced vital capacity increases with increasing body mass index: a paradoxical difference in low and normal body mass indices. Respir. Care 60, 113-118, https://doi.org/10.4187/ respcare.03403 (2015).

32. Chhabra, S. K. Forced vital capacity, slow vital capacity, or inspiratory vital capacity: which is the best measure of vital capacity? J. Asthma 35, 361-365, https://doi.org/10.3109/02770909809075669 (1998).

33. Galesanu, R. G. et al. Obesity in chronic obstructive pulmonary disease: is fatter really better? Can. Respir. J. 21, 297-301, https://doi. org/10.1155/2014/181074(2014).

34. Poulain, M. et al. The effect of obesity on chronic respiratory diseases: pathophysiology and therapeutic strategies. CMAJ 174, 1293-1299, https://doi.org/10.1503/cmaj.051299 (2006).

35. O'Donnell, D. E. et al. Effects of BMI on static lung volumes in patients with airway obstruction. Chest 140, 461-468, https://doi. org/10.1378/chest.10-2582 (2011).

36. Bhatt, S. P. et al. Discriminative Accuracy of FEV1:FVC Thresholds for COPD-Related Hospitalization and Mortality. JAMA 321, 2438-2447, https://doi.org/10.1001/jama.2019.7233 (2019).

37. Aryal, S., Diaz-Guzman, E. \& Mannino, D. M. COPD and gender differences: an update. Transl. Res. 162, 208-218, https://doi. org/10.1016/j.trsl.2013.04.003 (2013).

38. Aryal, S., Diaz-Guzman, E. \& Mannino, D. M. Influence of sex on chronic obstructive pulmonary disease risk and treatment outcomes. Int. J. Chron. Obstruct. Pulmon. Dis. 9, 1145-1154, https://doi.org/10.2147/COPD.S54476 (2014). 
39. Petersen, H. et al. The Course of Lung Function in Middle-aged Heavy Smokers: Incidence and Time to Early Onset of Chronic Obstructive Pulmonary Disease. Am. J. Respir. Crit. Care Med. 198, 1449-1451, https://doi.org/10.1164/rccm.201805-0861LE (2018).

40. Lange, P. et al. Lung-Function Trajectories Leading to Chronic Obstructive Pulmonary Disease. N. Engl. J. Med. 373, 111-122, https://doi.org/10.1056/NEJMoa1411532 (2015).

41. Cerveri, I. et al. The rapid FEV(1) decline in chronic obstructive pulmonary disease is associated with predominant emphysema: a longitudinal study. COPD 10, 55-61, https://doi.org/10.3109/15412555.2012.727920 (2013).

42. Bowler, R. P. et al. Prediction of acute respiratory disease in current and former smokers with and without COPD. Chest 146, 941-950, https://doi.org/10.1378/chest.13-2946 (2014).

43. Dransfield, M. T. et al. Acute Exacerbations and Lung Function Loss in Smokers with and without Chronic Obstructive Pulmonary Disease. Am. J. Respir. Crit. Care Med. 195, 324-330, https://doi.org/10.1164/rccm.201605-1014OC (2017).

44. Singh, S. J. et al. An official systematic review of the European Respiratory Society/American Thoracic Society: measurement properties of field walking tests in chronic respiratory disease. Eur. Respir. J. 44, 1447-1478, https://doi. org/10.1183/09031936.00150414 (2014)

45. Prahm, K. P., Witting, N. \& Vissing, J. Decreased variability of the 6-minute walk test by heart rate correction in patients with neuromuscular disease. PLoS One 9, e114273, https://doi.org/10.1371/journal.pone.0114273 (2014).

46. Martinez, F. J. et al. Predictors of mortality in patients with emphysema and severe airflow obstruction. Am. J. Respir. Crit. Care Med. 173, 1326-1334, https://doi.org/10.1164/rccm.200510-1677OC (2006).

47. Zeng, S. et al. Lung volume indices predict morbidity in smokers with preserved spirometry. Thorax 74, 114-124, https://doi. org/10.1136/thoraxjnl-2018-211881 (2019).

\section{Acknowledgements}

This study was supported by Award Number U01 HL089897 and Award Number U01 HL089856 from the National Heart, Lung, and Blood Institute. The content is solely the responsibility of the authors and does not necessarily represent the official views of the National Heart, Lung, and Blood Institute or the National Institutes of Health. Spyridon fortis was supported by the Department of Veterans Affairs, Veterans Health Administration, Office of Rural Health, Veterans Rural Health Resource Center (Award \# 14380), and the Health Services Research and evelopment (HSR\&D) Service through the Comprehensive Access and Delivery Research and Evaluation (CADRE) Center (CIN 13-412). Emily Wan was supported by Veterans Affairs Rehabilitation Research and Development Grant IK2RX002165.

\section{Author contributions}

S.F. is the guarantor of the study, had full access to all of the data in the study and takes responsibility for the integrity of the data and the accuracy of the data analysis. S.F. and E.S.W. participated in study conception and design, data analysis and drafting of the manuscript. S.F., A.C., V.K., R.C., J.E.H., J.D.C., E.K.S. and E.S.W. participated in data interpretation and critical revision of the manuscript.

\section{Competing interests}

In the past three years, Edwin K. Silverman received honoraria from Novartis for Continuing Medical Education Seminars and grant and travel support from GlaxoSmithKline. The rest of authors declare no competing interests.

\section{Additional information}

Supplementary information is available for this paper at https://doi.org/10.1038/s41598-020-61932-0.

Correspondence and requests for materials should be addressed to S.F.

Reprints and permissions information is available at www.nature.com/reprints.

Publisher's note Springer Nature remains neutral with regard to jurisdictional claims in published maps and institutional affiliations.

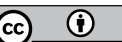

Open Access This article is licensed under a Creative Commons Attribution 4.0 International License, which permits use, sharing, adaptation, distribution and reproduction in any medium or format, as long as you give appropriate credit to the original author(s) and the source, provide a link to the Creative Commons license, and indicate if changes were made. The images or other third party material in this article are included in the article's Creative Commons license, unless indicated otherwise in a credit line to the material. If material is not included in the article's Creative Commons license and your intended use is not permitted by statutory regulation or exceeds the permitted use, you will need to obtain permission directly from the copyright holder. To view a copy of this license, visit http://creativecommons.org/licenses/by/4.0/.

(C) The Author(s) 2020 